\title{
Modeling of the Primary Acts of the Interaction between a Cell and an External Mechanical Field
}

\author{
Irina V. Ogneva ${ }^{1,2^{*}}$, Maria A. Usik ${ }^{1,2}$, Nikolay S. Biryukov ${ }^{1,2}$, Nikita O. Kremenetskii ${ }^{3}$, \\ Yuliya S. Zhdankina ${ }^{2}$
}

${ }^{1}$ Cell Biophysics Laboratory, State Scientific Center of the Russian Federation Institute of Biomedical Problems of the Russian Academy of Sciences, Moscow, Russia

${ }^{2}$ I. M. Sechenov First Moscow State Medical University, Moscow, Russia

${ }^{3}$ Ishlinsky Institute for Problems in Mechanics of the Russian Academy of Sciences, Moscow, Russia

Email: *iogneva@yandex.ru

How to cite this paper: Ogneva, I.V., Usik, M.A., Biryukov, N.S., Kremenetskii, N.O. and Zhdankina, Y.S. (2019) Modeling of the Primary Acts of the Interaction between a Cell and an External Mechanical Field. Applied Mathematics, 10, 527-544. https://doi.org/10.4236/am.2019.107037

Received: July 2, 2019

Accepted: July 16, 2019

Published: July 19, 2019

Copyright $\odot 2019$ by author(s) and Scientific Research Publishing Inc. This work is licensed under the Creative Commons Attribution International License (CC BY 4.0).

http://creativecommons.org/licenses/by/4.0/

\begin{abstract}
The mechanism of interaction between a cell and an external mechanical field is still poorly understood, and the accumulated diverse experimental data are often scattered. Therefore, the aim of this work was to systematize the experimental data in a mathematical model of the interaction between a cell and an external mechanical field based on standard kinetic equations and Fick's diffusion equation. Assuming that the cortical cytoskeleton proteins play a key role in cell mechanosensitivity, we compared the results of mathematical modeling and experimental data concerning the content of cytoskeletal proteins at the early stages of a mechanical field change. In addition, the proposed mathematical model suggests the dynamics of changes of a key transcription factor, which is necessary for the expression of certain genes encoding cytoskeletal proteins.
\end{abstract}

\section{Keywords}

Cell Mechanosensititvity, Kinetic Equations, Cortical Cytoskeleton

\section{Introduction}

Human exploration of outer space faces a number of unsolved problems, including medical problems. Being in conditions of weightlessness, even during Earth orbit, leads to a number of negative effects, for example, on the musculoskeletal and cardiovascular systems [1] [2] [3]. Existing methods of countermeasures, for the most part, are palliative, which is associated with a lack of un- 
derstanding of the etiology of the development of hypo-gravitational changes at the cellular and molecular levels. To date, questions remain about the interaction of a cell and a gravitational field: what is the mechanosensor and how the mechanotransduction paths are started.

Practically every intracellular structure can claim to be a mechanosensor. Thus, stretching of neurons or smooth muscle cells in a culture through the extracellular matrix leads to an increase in microtubule polymerization [4] [5]. Direct stretching of cell membranes, for example, using patch clamp technology, changes the cation-transport activity of mechanosensitive ion channels as a result of conformational changes of either the lipid bilayer [6] [7] or the portal domains of the channel itself [8] [9]. In addition, the submembrane cytoskeleton [10], as well as intracellular structures [11] [12], can also act as a mechanosensor.

The result of mechanotransduction is the formation of an adaptation pattern of proteins and gene expression. Thus, in cultured cells under conditions of altered gravity, there was a change in the cell profile, disorganization of microfilaments and, sometimes, microtubules [13]-[18], and changes in mitochondrial localization [19], which is determined by the state of the intermediate filaments. In addition, the changes are not limited to the protein content but also occur at the level of the expression of genes encoding cytoskeletal proteins and associated components of signaling cascades [20]-[26].

Our previous studies have suggested the role of the actin-binding proteins of the submembrane cytoskeleton in the primary mechanoreception of cells of various types, in particular skeletal muscle and myocardium. We assume that any change in the external conditions for the cell is reflected in the deformation of its cortical cytoskeleton. However, these strains are fundamentally different with increasing and decreasing loads. The first result is the dissociation of various actin-binding proteins from the cortical cytoskeleton: alpha-actinin-4 with a load decrease and alpha-actinin-1 with an increase [10] [27] [28]. With further development of this process, the deformation leads to the destruction of the structure and, at subsequent early stages of exposure, to an initial decrease in stiffness, which correlates with the content of actin non-muscle isoforms in the membrane fraction, which form the cortical cytoskeleton [29]. Furthermore, in the case of a decrease in external mechanical stress, there is a decrease in the expression of genes encoding cytoskeletal proteins and a further decrease in cortical cytoskeleton stiffness. In contrast, an increase in the external mechanical stress increases the mRNA content of the genes encoding cytoskeletal proteins and proteins directly and increases the stiffness [30] [31]. In general, the process of sensitivity to external stress by cells can be quite universal in the evolutionary series. Thus, Drosophila melanogaster lacks the isoform alpha-actinin-4; however, it is possible that supervillin plays a role in the process of mechanosensitivity [32].

Thus, the multiple components and variability of the mechanisms of cellular mechanosensitivity and mechanotransduction make it difficult to find the "hot 
spots" of its regulation and, as a consequence, the development of effective protection methods. Therefore, we decided to build a mathematical model based on the classical kinetic equations of the concentrations of key proteins and mRNAs involved in the perception and transduction of external mechanical stress, taking into account their diffusion between the compartments.

\section{Formulation of the Problem}

Based on the experimental results described above, it is possible to suggest the following mechanism for triggering the formation of an adaptive response to changes in the external mechanical stress.

Suppose that a sensitive protein $S P$ associated with microfilaments reacts to any change in mechanical stress. In addition, its antagonist, $a S P$, is also associated with microfilaments. Protein $S P$ can exist both in connection with microfilaments and in free form. The binding to the microfilament network and dissociation from microfilaments at the initial state occur to maintain the initial level of free protein $S P_{\text {free }}$ in both membrane $S P_{\text {freem } 0}$ and cytoplasmic compartment $S P_{\text {freec } 0}$. Free-form $S P_{\text {free }}$ can diffuse between compartments.

A change in the external mechanical stress leads to an increase in the content of free "sensitive" protein in the membrane fraction $S P_{\text {freem }}$ with a reaction rate constant, which depends both on the external mechanical stress and on the membrane compartment characteristics $v_{M F m \rightarrow S P f r e e m}^{*}\left(g, z_{m c}\right)$. An increase in the content of free $S P_{\text {freem }}$ in the membrane leads to an increase in its content in the cytoplasm $S P_{\text {freec }}$ due to diffusion. $S P_{\text {freec }}$ activates some protein $M_{c}^{*}$. The activated protein $M_{c}^{*}$ in turn activates the transcription factor $T F_{c}^{*}$ to diffuse into the nucleus and alter the transcription efficiency of its target genes and the formation of the corresponding mRNA. There is feedback, and the efficiency of the formation of the "sensitive" protein mRNA, rSP, depends on the content of activated $T F_{n}^{*}$ in the nucleus.

Suppose also that the efficiency of proteolysis and degradation of mRNA does not depend on the content and type of substrates and the rates of cleavage are constant $-v_{p}$ and $v_{d}$ for proteins and mRNA, respectively. In general, we assume that all reactions proceed at a constant rate, bearing in mind that the rate does not depend on the content of the substrate/reaction product.

We introduce the following notation:

$t$-time, index 0 - the initial moment of time;

the indices $m$ and $c$-the membrane and cytoplasmic compartments, respectively;

$z_{m c}$-the coordinate perpendicular to the cross section of the cell between the membrane and cytoplasmic compartments, along which the sensitive protein, its antagonist and the cytoskeletal proteins undergo diffusion,

$z_{c n}$-similarly, the coordinate perpendicular to the cell cross section between the cytoplasmic and nuclear compartments, along which the transcription factor is diffused; 
$D_{S P}$-the diffusion coefficient of the molecules of the "sensitive" protein between the cell compartments;

$D_{a S P}$-the diffusion coefficient of antagonist molecules of the "sensitive" protein between the cell compartments;

$S P_{\text {free }}$ - the content of the "sensitive" protein in the free state;

$a S P_{\text {free }}$ - the content of the antagonist of the "sensitive" protein in a free state;

$v_{M F \rightarrow \text { SPfree }}^{*}\left(g, z_{m c}\right)$-reaction rate of the transition of the "sensitive" protein from the complex with microfilaments to the free state when the mechanical stress is changed,

$r S P$ - the content of the "sensitive" protein mRNA;

raSP - the content of the antagonist of the "sensitive" protein mRNA;

$v_{d}$-the reaction rate of mRNA degradation in the cytoplasm;

$v_{S P \text { freec }}(r S P)$-the reaction rate of the synthesis of sensitive protein molecules, depending on the content of the corresponding mRNA $r S P$;

$v_{\text {aSfreec }}(\mathrm{raSP})$ - the reaction rate of the synthesis of antagonist molecules of the "sensitive" protein, depending on the content of the corresponding mRNA raSP;

$v_{p}$-is the reaction rate of proteolysis of protein molecules in the cytoplasm;

$M_{c}^{*}$ - the content of the activated modifying factor in the cytoplasm;

$v_{M_{c}^{*}}\left(S P_{\text {freec }}\right)$-the reaction rate of the activation of the modifying protein depending on the content of the free "sensitive" protein in the cytoplasm $S P_{\text {freec }}$;

$T F^{*}$-activated transcription factor;

$v_{T F_{c}^{*}}\left(M_{c}^{*}\right)$-reaction rate of formation of an activated transcription factor in the cytoplasm, depending on the content of the activated modifying protein $M_{c}^{*}$;

$v_{r S P s}\left(T F_{n}^{*}\right)$-the reaction rate of the synthesis of mRNA molecules of the "sensitive" protein, depending on the content of the activated transcription factor in the nucleus $T F_{n}^{*}$.

$S P$-the content of "sensitive" protein, which was evaluated experimentally;

$a S P$ - the content of the antagonist "sensitive" protein, which was estimated experimentally;

$M F$ - the content of major proteins that form microfilaments (actin isoforms);

$M T$ - the content of the main proteins forming microtubules (tubulin);

$I F$ - the content of basic proteins forming intermediate filaments (desmin);

$D_{M F}$-diffusion coefficient of microfilament monomer molecules between cell compartments;

$D_{M T}$-the diffusion coefficient of microtubule monomer molecules between cell compartments;

$D_{I F}$-diffusion coefficient of monomer molecules of intermediate filaments between the cell compartments;

$r M F$ - the microfilament mRNA content;

$r M T$ - the microtubule mRNA content;

$r I F$ - the intermediate filament mRNA content; 
$v_{\text {aSPrreec }}(\mathrm{raSP})$-the reaction rate of the synthesis of antagonist molecules of the "sensitive" protein, depending on the content of the corresponding mRNA raSP;

$v_{M F c}(r M F)$ - the reaction rate of the synthesis of microfilament molecules, depending on the content of the corresponding mRNA $r M F$;

$v_{M T C}(r M T)$-the reaction rate of synthesis of microtubule molecules, depending on the content of the corresponding mRNA $r M T$;

$v_{I F c}(r I F)$ - the reaction rate of synthesis of molecules of intermediate filaments, depending on the content of the corresponding mRNA $r I F$.

Then, for the proposed mechanism, the standard kinetic dependencies taking into account the diffusion between the compartments, the efficiency of synthesis and degradation for the concentrations of the analyzed proteins and mRNA are:

$$
\begin{aligned}
& S P_{\text {freem }}=S P_{\text {freem } 0}+D_{S P} \frac{\mathrm{d} S P_{\text {freec }}}{\mathrm{d} z_{m c}} \cdot t-D_{S P} \frac{\mathrm{d} S P_{\text {freem }}}{\mathrm{d} z_{m c}} \cdot t+v_{M F m \rightarrow S P \text { freem }}^{*}\left(g, z_{m c}\right) \cdot t \\
& S P_{\text {freec }}=S P_{\text {freec } 0}+v_{S P f r e e c}(r S P) \cdot t-v_{p} \cdot t-D_{S P} \frac{\mathrm{d} S P_{\text {freec }}}{\mathrm{d} z_{m c}} \cdot t+D_{S P} \frac{\mathrm{d} S P_{\text {freem }}}{\mathrm{d} z_{m c}} \cdot t \\
& M_{c}^{*}=M_{c 0}^{*}+v_{M_{c}^{*}}\left(S P_{f r e e c}\right) \cdot t-v_{p} \cdot t \\
& T F_{c}^{*}=T F_{c 0}^{*}+v_{T F_{c}^{*}}\left(M_{c}^{*}\right) \cdot t-D_{T F} \frac{\mathrm{d} T F_{c}^{*}}{\mathrm{~d} z_{c n}} t-v_{p} \cdot t \\
& r S P=r S P_{0}+v_{r S P S}\left(T F_{n}^{*}\right) \cdot t-v_{d} \cdot t \\
& T F_{n}^{*}=T F_{n 0}^{*}+D_{T F} \frac{\mathrm{d} T F_{c}^{*}}{\mathrm{~d} z_{c n}} t-v_{p} \cdot t \\
& a S P_{\text {freem }}=a S P_{\text {freem } 0}+D_{a S P} \frac{\mathrm{d} a S P_{\text {freec }}}{\mathrm{d} z_{m c}} \cdot t-D_{a S P} \frac{\mathrm{d} a S P_{\text {freem }}}{\mathrm{d} z_{m c}} \cdot t \\
& a S P_{\text {freec }}=a S P_{\text {freec } 0}+v_{a S P f r e e c}(r a S P) \cdot t-v_{p} \cdot t-D_{a S P} \frac{\mathrm{d} a S P_{\text {freec }}}{\mathrm{d} z_{m c}} \cdot t \\
& +D_{a S P} \frac{\mathrm{d} a S P_{\text {freem }}}{\mathrm{d} z_{m c}} \cdot t
\end{aligned}
$$

Similar to the previous system, for comparison with the experimental results and to determine the type of dependencies, we write the expressions for those parameters that can be determined (divided into compartments):

Membrane + cortical cytoskeleton

$$
\begin{gathered}
S P_{m}=S P_{m 0}+D_{S P} t\left(\frac{\mathrm{d} S P_{\text {freec }}}{\mathrm{d} z_{m c}}-\frac{\mathrm{d} S P_{\text {freem }}}{\mathrm{d} z_{m c}}\right) \\
a S P_{m}=a S P_{m 0}+D_{a S P} t\left(\frac{\mathrm{d} a S P_{\text {freec }}}{\mathrm{d} z_{m c}}-\frac{\mathrm{d} a S P_{\text {freem }}}{\mathrm{d} z_{m c}}\right) \\
M F_{m}=M F_{m 0}+D_{M F} t\left(\frac{\mathrm{d} M F_{c}}{\mathrm{~d} z_{m c}}-\frac{\mathrm{d} M F_{m}}{\mathrm{~d} z_{m c}}\right)
\end{gathered}
$$




$$
\begin{gathered}
M T_{m}=M T_{m 0}+D_{M T} t\left(\frac{\mathrm{d} M T_{c}}{\mathrm{~d} z_{m c}}-\frac{\mathrm{d} M T_{m}}{\mathrm{~d} z_{m c}}\right) \\
I F_{m}=I F_{m 0}+D_{I F} t\left(\frac{\mathrm{d} I F_{c}}{\mathrm{~d} z_{m c}}-\frac{\mathrm{d} I F_{m}}{\mathrm{~d} z_{m c}}\right)
\end{gathered}
$$

Cytoplasm

$$
\begin{gathered}
S P_{c}=S P_{c 0}+v_{S P f r e e c}(r S P) \cdot t-v_{p} \cdot t-D_{S P} t\left(\frac{\mathrm{d} S P_{f r e e c}}{\mathrm{~d} z_{m c}}-\frac{\mathrm{d} S P_{\text {freem }}}{\mathrm{d} z_{m c}}\right) \\
a S P_{c}=a S P_{c 0}+v_{a S P f r e e c}(r a S P) \cdot t-v_{p} \cdot t-D_{a S P} t\left(\frac{\mathrm{d} a S P_{f r e e c}}{\mathrm{~d} z_{m c}}-\frac{\mathrm{d} a S P_{f r e e m}}{\mathrm{~d} z_{m c}}\right) \\
M F_{c}=M F_{c 0}+v_{M F c}(r M F) \cdot t-v_{p} \cdot t-D_{M F} t\left(\frac{\mathrm{d} M F_{c}}{\mathrm{~d} z_{m c}}-\frac{\mathrm{d} M F_{m}}{\mathrm{~d} z_{m c}}\right) \\
M T_{c}=M T_{c 0}+v_{M T c}(r M T) \cdot t-v_{p} \cdot t-D_{M T} t\left(\frac{\mathrm{d} M T_{c}}{\mathrm{~d} z_{m c}}-\frac{\mathrm{d} M T_{m}}{\mathrm{~d} z_{m c}}\right) \\
I F_{c}=I F_{c 0}+v_{I F c}(r I F) \cdot t-v_{p} \cdot t-D_{I F} t\left(\frac{\mathrm{d} I F_{c}}{\mathrm{~d} z_{m c}}-\frac{\mathrm{d} I F_{m}}{\mathrm{~d} z_{m c}}\right) \\
r S P=r S P_{0}+v_{r S P_{s}}\left(T F_{n}^{*}\right) \cdot t-v_{d} \cdot t \\
r a S P=r a S P_{0}+v_{r a S P s}\left(T F_{n}^{*}\right) \cdot t-v_{d} \cdot t \\
r M F=r M F_{0}+v_{r M F s}\left(T F_{n}^{*}\right) \cdot t-v_{d} \cdot t \\
r M T=r M T_{0}+v_{r M T s}\left(T F_{n}^{*}\right) \cdot t-v_{d} \cdot t \\
r I F F_{0}+v_{r I F s}\left(T F_{n}^{*}\right) \cdot t-v_{d} \cdot t
\end{gathered}
$$

Solving equations together, we obtain expressions for the content of various proteins. For the modifying protein and transcription factor:

$$
\begin{gathered}
M_{c}^{*}=M_{c 0}^{*}+v_{M_{c}^{*}}\left(S P_{f r e e c}\right) \cdot t-v_{p} \cdot t \\
T F_{c}^{*}=T F_{c 0}^{*}-v_{p} \cdot t+v_{T F_{c}^{*}}\left(M_{c}^{*}\right) \cdot t-\left(T F_{c 0}^{*}-v_{p} \cdot t+v_{T F_{c}^{*}}\left(M_{c}^{*}\right) \cdot t\right) \cdot \mathrm{e}^{-\frac{z_{c n}}{D_{T F} t}} \\
T F_{n}^{*}=T F_{n 0}^{*}-v_{p} \cdot t+\left(T F_{c 0}^{*}-v_{p} \cdot t+v_{T F_{c}^{*}}\left(M_{c}^{*}\right) \cdot t\right) \cdot \mathrm{e}^{-\frac{z_{c n}}{D_{T F} t}}
\end{gathered}
$$

For “sensitive" protein:

$$
\begin{gathered}
r S P=r S P_{0}+v_{r S P S}\left(T F_{n}^{*}\right) \cdot t-v_{d} \cdot t \\
S P_{\text {freec }}=S P_{\text {freec } 0}-v_{p} \cdot t+v_{S P_{\text {freec }}}(r S P) \cdot t \\
-\left(S P_{\text {freec } 0}-v_{p} \cdot t+v_{S P_{\text {freec }}}(r S P) \cdot t\right) \cdot \mathrm{e}^{-\frac{z_{m c}}{2 D_{S P} t}} \\
+\frac{\mathrm{e}^{-\frac{z_{m c}}{2 D_{S P} t}}}{2 D_{S P}} \int_{0}^{z_{m c}} \frac{\mathrm{d} k_{M F m \rightarrow S P f r e e m}^{*}\left(g, z_{m c}\right)}{\mathrm{d} z_{m c}} \cdot \mathrm{e}^{\frac{z_{m c}}{2 D_{S P} t}} \mathrm{~d} z_{m c}
\end{gathered}
$$




$$
\begin{aligned}
S P_{\text {freem }}= & S P_{\text {freem } 0}+v_{M F m \rightarrow S P f r e e m}^{*}\left(g, z_{m c}\right) \cdot t \\
& +\left(S P_{\text {freec } 0}-v_{p} \cdot t+v_{S P_{\text {freec }}}(r S P) \cdot t\right) \cdot \mathrm{e}^{-\frac{z_{m c}}{2 D_{S P} t}} \\
& -\frac{\mathrm{e}^{-\frac{z_{m c}}{2 D_{S P} t}}}{2 D_{S P}} \int_{0}^{z_{m c}} \frac{\mathrm{d} k_{M F m \rightarrow S P f r e e m}^{*}\left(g, z_{m c}\right)}{d z_{m c}} \cdot \mathrm{e}^{\frac{z_{m c}}{2 D_{S P} t}} \mathrm{~d} z_{m c} \\
S P_{c}= & S P_{c 0}+\left(S P_{\text {freec } 0}-v_{p} \cdot t+v_{S P_{\text {freec }}}(r S P) \cdot t\right) \cdot \mathrm{e}^{-\frac{z_{m c}}{2 D_{S P} t}} \\
& +t \cdot \frac{\mathrm{d}}{\mathrm{d} z_{m c}}\left(\mathrm{e}^{-\frac{z_{m c}}{2 D_{S P} t}} \cdot \int_{0}^{z_{m c}} \frac{\mathrm{d} k_{M F m \rightarrow S P \text { freem }}^{*}\left(g, z_{m c}\right)}{\mathrm{d} z_{m c}} \cdot \mathrm{e}^{\frac{z_{m c}}{2 D_{S P} t}} \mathrm{~d} z_{m c}\right) \\
S P_{m}= & S P_{m 0}-v_{p} \cdot t+v_{S P_{\text {freec }}}(r S P) \cdot t \\
& -\left(S P_{\text {freec } 0}-v_{p} \cdot t+v_{S P_{\text {freec }}}(r S P) \cdot t\right) \cdot \mathrm{e}^{-\frac{z_{m c}}{2 D_{S P} t}} \\
& -t \cdot \frac{\mathrm{d}}{\mathrm{d} z_{m c}}\left(\mathrm{e}^{-\frac{z_{m c}}{2 D_{S P} t}} \cdot \int_{0}^{z_{m c}} \frac{\mathrm{d} k_{M F m \rightarrow S P \text { freem }}^{*}\left(g, z_{m c}\right)}{\mathrm{d} z_{m c}} \cdot \mathrm{e}^{\frac{z_{m c}}{2 D_{S P t}} \mathrm{~d} z_{m c}}\right)
\end{aligned}
$$

For the antagonist of "sensitive" protein:

$$
\begin{gathered}
r a S P=r a S P_{0}+v_{\text {raSPs }}\left(T F_{n}^{*}\right) \cdot t-v_{d} \cdot t \\
a S P_{\text {freec }}=a S P_{\text {freec } 0}-v_{p} \cdot t+v_{a S P_{\text {freec }}}(r a S P) \cdot t \\
-\left(a S P_{\text {freec } 0}-v_{p} \cdot t+v_{a S P_{\text {freec }}}(\mathrm{raSP}) \cdot t\right) \cdot \mathrm{e}^{-\frac{z_{m c}}{2 D_{a S P} t}} \\
a S P_{\text {freem }}=a S P_{\text {freem } 0}+\left(a S P_{\text {freec } 0}-v_{p} \cdot t+v_{a S P_{\text {freec }}}(\mathrm{raSP}) \cdot t\right) \cdot \mathrm{e}^{-\frac{z_{m c}}{2 D_{a S P} t}} \\
a S P_{c}=a S P_{c 0}-v_{p} \cdot t+v_{a S P_{\text {freec }}}(\mathrm{raSP}) \cdot t \\
-\left(a S P_{\text {freec } 0}-v_{p} \cdot t+v_{a S P_{\text {freec }}}(\mathrm{raSP}) \cdot t\right) \cdot \mathrm{e}^{-\frac{z_{m c}}{2 D_{a S P} t}} \\
a S P_{m}=a S P_{m 0}+\left(a S P_{\text {freec } 0}-v_{p} \cdot t+v_{a S P_{\text {freec }}}(\mathrm{raSP}) \cdot t\right) \cdot \mathrm{e}^{-\frac{z_{m c}}{2 D_{a S P} t}}
\end{gathered}
$$

For microfilaments:

$$
\begin{gathered}
r M F=r M F_{0}+v_{r M F s}\left(T F_{n}^{*}\right) \cdot t-v_{d} \cdot t \\
M F_{c}=M F_{c 0}-v_{p} \cdot t+v_{M F c}(r M F) \cdot t \\
-\left(M F_{c 0}-v_{p} \cdot t+v_{M F c}(r M F) \cdot t\right) \cdot \mathrm{e}^{-\frac{z_{m c}}{2 D_{M F} t}} \\
M F_{m}=M F_{m 0}+\left(M F_{c 0}-v_{p} \cdot t+v_{M F c}(r M F) \cdot t\right) \cdot \mathrm{e}^{-\frac{z_{m c}}{2 D_{M F} t}}
\end{gathered}
$$

For microtubules:

$$
\begin{array}{rl}
r & M T=r M T_{0}+v_{r M T s}\left(T F_{n}^{*}\right) \cdot t-v_{d} \cdot t \\
M T_{c}= & M T_{c 0}-v_{p} \cdot t+v_{M T c}(r M T) \cdot t \\
& -\left(M T_{c 0}-v_{p} \cdot t+v_{M T c}(r M T) \cdot t\right) \cdot \mathrm{e}^{-\frac{z_{m c}}{2 D_{M T} t}}
\end{array}
$$




$$
M T_{m}=M T_{m 0}+\left(M T_{c 0}-v_{p} \cdot t+v_{M T c}(r M T) \cdot t\right) \cdot \mathrm{e}^{-\frac{z_{m c}}{2 D_{M T} t}}
$$

For intermediate filaments:

$$
\begin{gathered}
r I F=r I F_{0}+v_{r I F s}\left(T F_{n}^{*}\right) \cdot t-v_{d} \cdot t \\
I F_{c}=I F_{c 0}-v_{p} \cdot t+v_{I F c}(r I F) \cdot t-\left(I F_{c 0}-v_{p} \cdot t+v_{I F c}(r I F) \cdot t\right) \cdot \mathrm{e}^{-\frac{z_{m c}}{2 D_{I F} t}} \\
I F_{m}=I F_{m 0}+\left(I F_{c 0}-v_{p} \cdot t+v_{I F c}(r I F) \cdot t\right) \cdot \mathrm{e}^{-\frac{z_{m c}}{2 D_{I F} t}}
\end{gathered}
$$

\section{Simulation}

In previous studies, we obtained systematic data concerning the contents of various cytoskeletal proteins in the membrane and cytoplasmic fractions of rat soleus muscle fibers [28] [29]. Therefore, for the simulation, we consider this type of cell under changes in the external mechanical field.

We consider the actin-binding proteins alpha-actinin- 4 and alpha-actinin- 1 as a "sensitive" protein and its antagonist, respectively, and beta-actin as a protein of microfilaments of the submembrane cytoskeleton because its content dominates over the content of gamma-actin in this cell type [28] (Figure 1(a)).

As a result of a change in the external mechanical stress, an adaptive pattern is formed: in the case of an increase, the cytoskeleton becomes more developed, in the case of a decrease, vice versa. Consider the option of decreasing external mechanical stress (Figure 1(b)).

We will follow the "sensitive" protein, its antagonist, and microfilaments and compare the results of the simulation with the experimental data.

Since the experiment evaluated the relative contents of proteins and mRNA as a whole in compartments, then $z_{m c}$ is the "path length" between the cortical
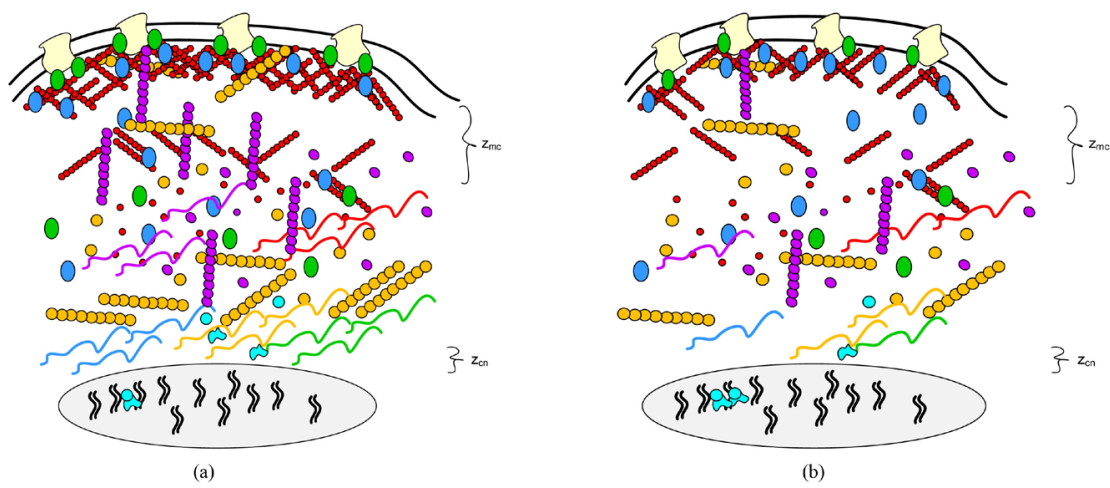

Figure 1. Schema of mechanosensitivity. (a) - an initial state; (b) - an adaptive pattern under decrease of the external mechanical tension. $\}$-cholesterol, $O-S P$, $\sim{ }_{-r S P}, O_{-a S P}, \sim{ }_{-r a S P},{ }_{0000000}-M F$ as filaments, $0-M F$ as monomers, $\mathcal{L}_{-r M F}, 00000000-M T$ as filaments, $\bigcirc-M T$ as monomers, $\sim-r M T, 00000000-I F$ as filaments, $\bigcirc-I F$ as monomers, $\sim-r I F$, $O-M_{c}^{*}, \cong-T F^{*}$. 
cytoskeleton and the cytoplasm, and $z_{c n}$ is the "path length" between the cytoplasm and chromatin (Figure 1).

We assume that for the fibers of the soleus muscle of rats:

$$
z_{m c}=18 \times 10^{-7} \mathrm{~m}, \quad z_{c n}=2 \times 10^{-7} \mathrm{~m} .
$$

Following [33], using the Stokes-Einstein equation, we assume that the diffusion coefficient is:

$$
D=\frac{R T}{6 \pi \eta r N_{A}},
$$

where $R=8.31 \mathrm{~J} / \mathrm{mol} \cdot \mathrm{K}$-the universal gas constant, $T=298 \mathrm{~K}$ - the temperature, $\quad \eta=10^{-3} \mathrm{~Pa} \cdot \mathrm{s}$ - the dynamic viscosity of the medium, $N_{A}=6.02 \times 10^{23} \mathrm{~mol}^{-1}, r-$ the hydrodynamic radius of the protein molecule.

Beta-actin, considered the main protein of microfilaments of the cortical cytoskeleton in rat soleus muscle, has 375 amino acid residues. Then, we will assume that its hydrodynamic radius is $5.28 \times 10^{-9} \mathrm{~m}$ [33]. Assuming that $S P$ and aSP are alpha-actinin-4 and alpha-actinin-1, having 911 and 892 amino acid residues, respectively, we will assume that for them, the hydrodynamic radii are the same and amount to $6.61 \times 10^{-9} \mathrm{~m}$, based on the extrapolation proposed by [33]. The transcription factor remains unknown in the proposed mechanism, but since many parameters are not determined accurately but are estimated, for simplicity of calculations, we will consider the hydrodynamic radius of the transcription factor as a certain average value, and we will use $6.0 \times 10^{-9} \mathrm{~m}$. Consequently, all the hydrodynamic radii necessary for calculations have close values, and we can assume that the diffusion coefficient has one order:

$$
D_{M F}=D_{S P}=D_{a S P}=D_{T F}=10^{-11} .
$$

The rate of RNA polymerase II in eukaryotic cells is 10 - 70 nucleotides per second [34] [35]. Thus, we defined it as 40 nucleotides/s. Since the dependence of the efficiency of the recruitment of a transcriptional complex to DNA in this case is unknown, we will assume that for each of the proteins under consideration, it is a linear relationship with a specific recruitment coefficient in each case. Then:

for $S P$ (alpha-actinin-4, $3885 \mathrm{bp})-v_{r S P S}\left(T F_{n}^{*}\right)=10^{-2} k_{r S P s} \cdot T F_{n}^{*} s^{-1}$;

for $a S P$ (alpha-actinin-1, $2956 \mathrm{bp})-v_{\text {raSPs }}\left(T F_{n}^{*}\right)=1.4 \times 10^{-2} k_{\text {raSPs }} \cdot T F_{n}^{*} s^{-1}$;

$$
\text { for } M F \text { (beta-actin, } 1293 \mathrm{bp})-v_{r M F}\left(T F_{n}^{*}\right)=3 \times 10^{-2} k_{r M F} \cdot T F_{n}^{*} s^{-1} \text {, }
$$

where $k_{r S P_{s}}, k_{r a S P_{s}}$ and $k_{r M F}$ are recruitment coefficients of the transcriptional complex to DNA depending on the content of the activated transcription factor in the nucleus for alpha-actinin-4, alpha-actinin- 1 and beta-actin, respectively.

We assume that the half-life of mRNA of genes encoding cytoskeletal proteins, as well as for globin, is approximately 8 hours [36]. We assume that on average, it is approximately 28,800 seconds. Therefore, the reaction rate constant for mRNA degradation in the cytoplasm is: 


$$
v_{d}=3.5 \times 10^{-5} \mathrm{~s}^{-1}
$$

The speed of ribosomes in eukaryotic cells is diverse, but we will assume that on average, including for cytoskeletal proteins, processing proceeds at a speed of 5 amino acid residues per second [37]. Then:

for $S P$ (alpha-actinin-4, 911aa) $-v_{S P \text { freec }}(r S P)=5.5 \times 10^{-3} k_{S P f r e e c} \cdot r S P S^{-1}$;

for aSP (alpha-actinin-1, 892aa) $-v_{\text {aSPfreec }}(\mathrm{raSP})=5.6 \times 10^{-3} k_{a S P f r e e c} \cdot \mathrm{raSPS}^{-1}$;

for $M F$ (beta-actin, 375aa) $-v_{M F c}(r M F)=13.3 \times 10^{-3} k_{M F c} \cdot r M F s^{-1}$

where the $k_{\text {SPfreec }}, k_{\text {aSPfreec }}$ and $k_{M F c}$ are coefficients that reflect the efficiency of translation for alpha-actinin-4, alpha-actinin-1 and beta-actin, respectively.

We assume that proteolysis is carried out using the proteasome. The rate of proteolysis depends on how long the protein has been synthesized but, on average, is 2.5 substrates/minute [38]. Let us assume that on average, for the analyzed proteins, the rate of proteolysis reaction of protein molecules in the cytoplasm is:

$$
v_{p}=4 \times 10^{-2} \mathrm{~s}^{-1}
$$

Since the dependence of the activation of the modifying protein on the content of free "sensitive" protein in the cytoplasm $S P_{\text {freec }}$ and the dependence of the formation of an activated transcription factor in the cytoplasm, which depend on the content of the activated modifying protein $M_{c}^{*}$, remain unknown, we approximate, as above, with the linear dependence specific activation factors:

$$
\begin{gathered}
v_{M_{c}^{*}}\left(S P_{\text {freec }}\right)=a_{M_{c}^{*}} \cdot S P_{\text {freec }} s^{-1} \\
v_{T F_{c}^{*}}\left(M_{c}^{*}\right)=a_{T F_{c}^{*}} \cdot M_{c}^{*} s^{-1}
\end{gathered}
$$

where $a_{M_{c}^{*}}$ and $a_{T F_{c}^{*}}$ are the activation coefficients of the modifying protein by the free "sensitive" protein and the transcription factor by the activated modified protein, respectively.

Consequently, the greatest uncertainty is $v_{M F \rightarrow \text { SPree }}^{*}\left(g, z_{m c}\right)$, the reaction rate of the transition of the "sensitive" protein from the complex with microfilaments to the free state when the mechanical stress changes. Without loss of generality, we will assume that the variables are independent and then:

$$
v_{M F \rightarrow \text { SPfree }}^{*}\left(g, z_{m c}\right)=v^{*}(g) \cdot v^{*}\left(z_{m c}\right)=v^{*}(g) \cdot D_{S P} \cdot t \cdot \frac{-\frac{z_{m c}}{2 D_{S P} t}}{}
$$

Considering gravity as a bulk force, we accept, as before [27], that:

$$
v^{*}(g)=\rho \cdot g \cdot \cos \varphi,
$$

where $\varphi$ is the orientation angle, in this case, the soleus muscle in the field of gravity, $g$ is the acceleration of gravity, and $\rho$ is the density of the cortical cytoskeleton, depending on the initial number of formed filaments and their organization into the network. For the standard model used to reproduce the effects of weightlessness on Earth in rodents, antiorthostatic suspension, this angle 
is $30^{\circ}$ [39].

We take the initial values of the estimated parameters for $100 \%$ and substitute (45) - (58) into (24) - (38). We evaluated the process of perception of a mechanical stimulus and its transduction at several points-6, 12, 18, 24 and 72 hours - to compare the results of mathematical modeling and experimental data obtained by us earlier [28].

The dependences obtained for the content of the "sensitive" proteinalpha-actinin-4 (Figure 2), the antagonist of the "sensitive" proteinalpha-actinin-1 (Figure 3), and microfilaments-beta-actin (Figure 4) in comparison with experimental data show coincidence when varying only the constants of the efficiency of transcription $\left(k_{r S P_{s}}, k_{r a S P_{s}}, k_{r M F}\right.$ respectively) and translation ( $k_{\text {SPfreec }}, k_{\text {aSPfreec }}, k_{M F c}$ respectively). However, there are differences at the 72 -hour point: in the experiment, the alpha-actinin- 1 content is $77 \% \pm 7 \%$ of the control in the membrane fraction and $50.5 \%$ in the numerical experiment (Figure 3(b)); in the experiment, the beta-actin content in the cytoplasmic fraction does not differ from the control; in the numerical experiment, it is reduced (66.4\% of the control) (Figure 4(c)). In addition, at the 12 o'clock point in the experiment, the beta-actin content in the membrane fraction is already reduced and amounts to $51 \% \pm 4 \%$ of the control; in a numerical experiment, it does not differ from the control (103\%) (Figure 4(b)).

The dynamics of the transcription factor change (Figure 5) indicates an increase in its content in the nucleus after 6 hours by 35\% and a subsequent increase up to 24 hours (235\% relative to the control) and then a decrease after 72 hours compared to the maximum accumulation (up to $190 \%$ ).

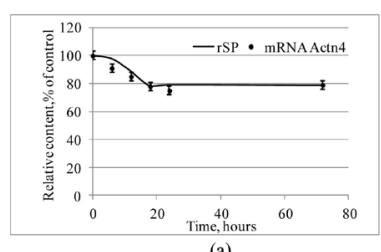

(a)

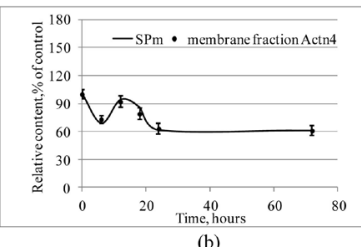

(b)

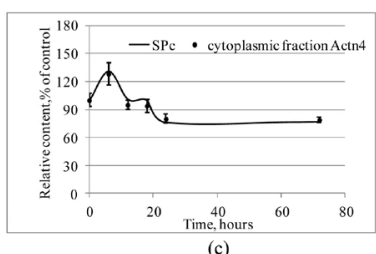

Figure 2. Relative content of the "sensitive" protein and its mRNA (simulation data) and comparison with the relative content of alpha-actinin-4 (Actn4) and mRNA (experimental data). (a) - mRNA comparison; (b) - membrane fraction of protein comparison; (c) - cytoplasmic fraction of protein comparison.

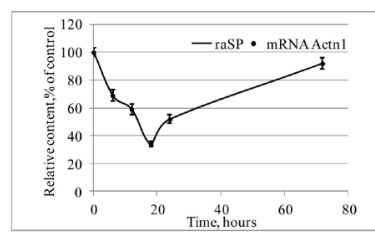

(a)

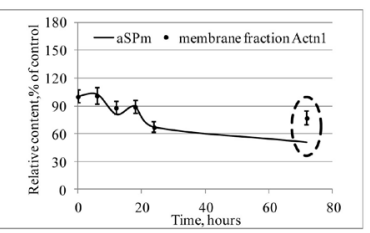

(b)

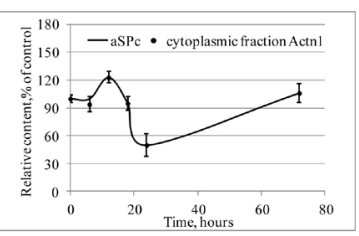

(c)

Figure 3. Relative content of the antagonist of the "sensitive" protein and its mRNA (simulation data) and comparison with the relative content of alpha-actinin-1 (Actn1) and mRNA (experimental data). (a) - mRNA comparison; (b) - membrane fraction of protein comparison; (c) - cytoplasmic fraction of protein comparison. 


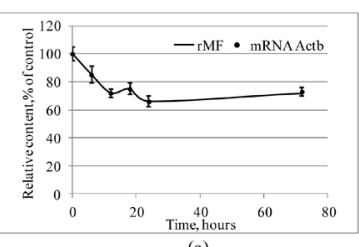

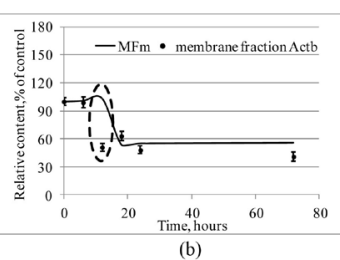

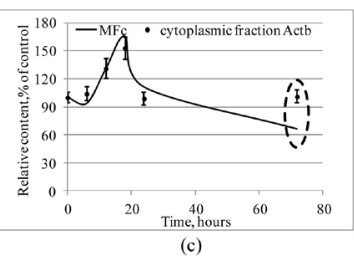

(c)

Figure 4. Relative content of microfilament protein and its mRNA (simulation data) and comparison with the relative content of beta-actin and mRNA (experimental data). (a) - mRNA comparison; (b) - membrane fraction of protein comparison; (c)cytoplasmic fraction of protein comparison.

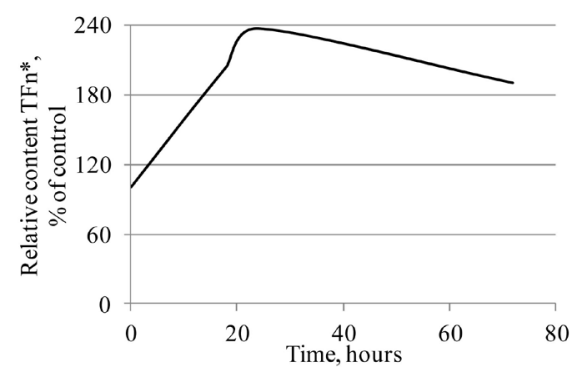

Figure 5. Relative content of the transcription factor in the nuclei (simulation data).

Simulation data were fitted by curve; experimental data are marked by dots. The simulation was performed under parameters (45) - (58) and constants for $6 \mathrm{~h} \quad k_{r S P s}=-5.28 \times 10^{-5}, \quad k_{S P f r e e c}=-18 \times 10^{-2}$, for $12 \mathrm{~h}-k_{r S P_{s}}=-4.4 \times 10^{-5}$, $k_{\text {SPfreec }}=-16 \times 10^{-2}$, for $18 \mathrm{~h}-k_{r S P_{s}}=-4.39 \times 10^{-5}, k_{S P \text { freec }}=-2 \times 10^{-2}$, for $24 \mathrm{~h}-$ $k_{r S P s}=3.36 \times 10^{-5}, \quad k_{S P f r e c}=-1.53 \times 10^{-3}, \quad$ for $72 \mathrm{~h}-k_{r S P s}=1.75 \times 10^{-5}$, $k_{\text {SPfreec }}=8.85 \times 10^{-4}$. The figure was built in the Excel 2007 for Windows.

Simulation data were fitted by curve; experimental data are marked by dots. The simulation was performed under parameters (45) - (58) and constants for $6 \mathrm{~h} \quad k_{r S P S}=-1.62 \times 10^{-4}, \quad k_{S P f r e c c}=10^{-4}$, for $12 \mathrm{~h}-k_{r S P s}=-1.11 \times 10^{-4}$, $k_{\text {SPfreec }}=2.23 \times 10^{-3}$, for $18 \mathrm{~h}-k_{r S P s}=2.84 \times 10^{-5}, k_{S P f r e e c}=2.01 \times 10^{-3}$, for 24 $\mathrm{h}-k_{r S P s}=-5.33 \times 10^{-5}, \quad k_{S P f r e e c}=-2.44 \times 10^{-3}$, for $72 \mathrm{~h}-k_{r S P s}=-5.33 \times 10^{-5}$, $k_{\text {SPfreec }}=-2.41 \times 10^{-3}$. The dotted ovals show differences between the simulation and experimental data. The figure was built in the Excel 2007 for Windows.

The simulation was performed under parameters (45) - (58). The figure was built in the Excel 2007 for Windows.

\section{Discussion}

The problem of perception by living cells of a mechanical stimulus is not only of practical importance associated with the exploration of deep space but also fundamental since life has evolved under the conditions of a permanently acting mechanical factor-gravity. Despite the many studies, there is currently no universal idea of how a cell perceives an external mechanical field and how it transduces its changes to form an adaptation pattern.

In our previous works, we obtained experimental data that suggest the key role of actin-binding proteins in the cells' perception of various types of me- 
chanical stimulus. For mammals, we assume that these proteins can be two calcium-dependent alpha-actinin forms: alpha-actinin-1 and alpha-actinin-4 [10] [27] [28]. However, for example, in Drosophila, there is only one alpha-actinin isoform, but our previous data suggest that another actin-binding protein, supervillin, may be the second participant [32]. Therefore, in this work, we designated this pair of proteins as a sensitive protein SP and an antagonist of the sensitive protein $a S P$.

In system biology, mathematical modeling is often used to estimate the values of unknown parameters. The model of population dynamics of Lotka-Volterra is used especially widely in various kinetic models, for example, when modeling the development of bacterial infection [40]. In this paper, the use of kinetic regularities and the second Fick's law, the diffusion equation, assuming that increasing or decreasing mechanical stress leads to dissociation of a $S P$ or $S P$ from the cortical cytoskeleton, let us numerically receive the same results as in the experiments.

We estimated the values of the parameters after 6, 12, 18, 24 and 72 hours. In almost all cases, by varying only the constants of the efficiency of transcription and translation, we obtained theoretical data that coincided with the experimental data. The exception was the content of the antagonist of the sensitive protein in the membrane fraction (alpha-actinin-1) after 72 hours of exposure: in the experiment, the minimum content was at the previous point, 24 hours [28], while modeling was at 72 hours. However, the error values of the experimental data do not show a significant difference between the values at 24 and 72 hours [28]. In addition, a significant difference between the experimental data and those obtained in a numerical experiment occurred in the membrane fraction of microfilaments after 12 hours, as well as in the cytoplasmic fraction, after 72 hours. However, it should be noted that in the experiment, we evaluated the content of actin isoforms (beta and gamma-) separately, while in a numerical experiment, for simplicity, we followed the total content of proteins forming microfilaments, comparing the data with beta-actin. However, because the content of beta-actin in muscle cells substantially dominates the content of gamma-actin, this approach can be justified. In general, this model, developed for early acts of cellular mechanoreception, gives results that coincide with experimental data up to 72 hours of exposure.

In addition, it is known that one of the candidates for the role of the sensitive protein $S P$, alpha-actinin-4, can penetrate into the nucleus [41] and bind to the promoter regions of the genes. However, it is still unknown, in the case of changes in external mechanical stress, whether alpha-actinin-4 itself regulates the expression of its potential targets or indirectly regulates them through a transcription factor. Therefore, we introduced a transcription factor modifier into the model, which can be activated by $S P$, and, in turn, activate the corresponding transcription factor. In a numerical experiment, the dynamics of changes in the content of the transcription factor in the nucleus were estimated, 
and according to these results, we can assume that the maximum of its accumulation occurs at 24 hours, where its content exceeds the control level by almost 2.5 times. In addition, its content in the nuclei begins to increase after 6 hours of exposure.

Alpha-actinin-4, according to experimental data, has approximately the same initial content in the membrane and cytoplasmic compartments. After 6 hours of exposure in the membrane compartment, its content decreases by $27 \%$ and increases in the cytoplasm by $30 \%$ [28] [29]. In the numerical experiment, we obtained the same data. However, at the same time, after 6 hours of exposure, according to the simulation data, the content of the activated transcription factor in the nucleus increases by $35 \%$. Comparing these data, we can assume that when the external mechanical stress changes, alpha-actinin-4 does not directly regulate the expression of the genes assessed but indirectly regulates them through the activation of an appropriate transcription factor.

Of course, such an assumption after mathematical modeling needs experimental verification, but it can be assumed that the disclosure of the detailed mechanism of interaction between the cell and the external mechanical field will help in the development of effective preventive measures that are necessary, for example, during deep space exploration.

\section{Conclusions}

The results obtained indicate that the model of perception of an external mechanical stimulus by living cells, based on a system of kinetic equations and the second Fick law, adequately describes the process, and the simulation results correlate with the experimental data.

The time dependencies estimated in a numerical experiment suggest that alpha-actinin-4 triggers a signaling cascade, leading to an increase in the content of certain transcription factors whose targets are both alpha-actinin-4 and alpha-actinin-1 and beta-actin. In other words, the answer to the question of whether alpha-actinin-4 regulates gene expression directly when mechanical stress changes (given its ability to penetrate into the nucleus and bind to some promoters) is as follows: according to a numerical experiment, it is more likely that another transcription factor will appear.

\section{Author Contributions}

Irina V. Ogneva-idea, conceptualization, data curation, formal analysis, funding acquisition, investigation, methodology, project administration, resources, software, supervision, validation, visualization, writing.

Maria A. Usik-formal analysis, investigation, methodology, visualization, writing.

Nikolay S. Biryukov-investigation, methodology, validation.

Nikita O. Kremenetsky-investigation, validation.

Yuliya S. Zhdankina-investigation, methodology, validation. 


\section{Funding}

This work was financially supported by the program for fundamental research SSC RF-IBMP RAS; program "Postgenomic technologies and perspective solutions in the biomedicine" of the RAS Presidium; Russian Academic Excellence Project 5-100.

\section{Disclosure of Potential Conflicts of Interest}

The authors declare that they have no conflict of interest.

\section{Data Availability Statement}

All data generated or analyzed during this study are included in this article.

\section{References}

[1] Riley, D.A., Bain, J.L.W., Thompson, J.L., Fitts, R.H., Widrick, J.J., Trappe, S.W. and Trappe, T.A. (2000) Decreased Thin Filament Density and Length in Human Atrophic Soleus Muscle fibers after Spaceflight. Journal of Applied Physiology, 88, 567-572. https://doi.org/10.1152/jappl.2000.88.2.567

[2] Vico, L. and Hargens, A. (2018) Skeletal Changes during and after Spaceflight. Nature Reviews Rheumatology, 14, 229-245. https://doi.org/10.1038/nrrheum.2018.37

[3] Watenpaugh, D.E. and Hargens, A.R. (1996) The Cardiovascular System in Microgravity. In: Handbook of Physiology. Environmental Physiology, Bethesda, MD, 631-674.

[4] Dennerll, T.J., Joshi, H.C., Steel, V.L., Buxbaum, R.E. and Heidemann, S.R. (1988) Tension and Compression in the Cytoskeleton of PC-12 Neurites. II: Quantitative Measurements. The Journal of Cell Biology, 107, 665-674. https://doi.org/10.1083/jcb.107.2.665

[5] Putnam, A.J., Schultz, K. and Mooney, D.J. (2001) Control of Microtubule Assembly by Extracellular Matrix and Externally Applied Strain. American Journal of Physiology, 280, C556-C564. https://doi.org/10.1152/ajpcell.2001.280.3.C556

[6] Sukharev, S. and Corey, D.P. (2004) Mechanosensitive Channels: Multiplicity of Families and Gating Paradigms. Science Signaling, 2004, re4. https://doi.org/10.1126/stke.2192004re4

[7] Maroto, R., Raso, A., Wood, T.G., Kurosky, A., Martinac, B. and Hamill, O.P. (2005) TRPC1 Forms the Stretch-Activated Cation Channel in Vertebrate Cells. Nature Cell Biology, 7, 179-185. https://doi.org/10.1038/ncb1218

[8] Sukharev, S., Betanzos, M., Chiang, C.S. and Guy, H.R. (2001) The Gating Mechanism of the Large Mechanosensitive Channel MscL. Nature, 409, 720-724. https://doi.org/10.1038/35055559

[9] Howard, J. and Bechstedt, S. (2004) Hypothesis: A Helix of Ankyrin Repeats of the NOMPC-TRP Ion Channel Is the Gating Spring of Mechanoreceptors. Current Biology, 14, R224-R226. https://doi.org/10.1016/j.cub.2004.02.050

[10] Ogneva, I.V. (2013) Cell Mechanosensitivity: Mechanical Properties and Interaction with Gravitational Field. BioMed Research International, 2013, Article ID: 598461. https://doi.org/10.1155/2013/598461

[11] Maniotis, A.J., Chen, C.S. and Ingber, D.E. (1997) Demonstration of Mechanical Connections between Integrins, Cytoskeletal Filaments and Nucleoplasm that Sta- 
bilize Nuclear Structure. Proceedings of the National Academy of Sciences of the United States of America, 94, 849-854. https://doi.org/10.1073/pnas.94.3.849

[12] Huang, H., Kamm, R.D. and Lee, R.T. (2004) Cell Mechanics and Mechanotransduction: Pathways, Probes, and Physiology. American Journal of Physiology-Cell Physiology, 287, C1-C11. https://doi.org/10.1152/ajpcell.00559.2003

[13] Sakar, D., Nagaya, T., Koga, K. and Seo, H. (1999) Culture in Vector-Averaged Gravity Environment in a Clinostat Results in Detachment of Osteoblastic ROS 17/2.8 Cells. Occupational and Environmental Medicine, 43, 22-24.

[14] Uva, B.M., Masini, M.A., Sturla, M., Prato, P., Passalacqua, M., Giuliani, M., Tagliafierro, G. and Strollo, F. (2002) Clinoration-Induced Weightlessness Influences the Cytoskeleton of Glial Cells in Culture. Brain Research, 934, 132-139. https://doi.org/10.1016/S0006-8993(02)02415-0

[15] Gaboyard, S., Blachard, M.P., Travo, B.T., Viso, M., Sans, A. and Lehouelleur, J. (2002) Weightlessness Affects Cytoskeleton of Rat Utricular Hair Cells during Maturation in Vitro. NeuroReport, 13, 2139-2142. https://doi.org/10.1097/00001756-200211150-00030

[16] Plett, P.A., Abonour, R., Frankovitz, S.M. and Orschell, C.M. (2004) Impact of Modeled Microgravity on Migration, Differentiation and Cell Cycle Control of Primitive Human Hematopoietic Progenitor Cells. Experimental Hematology, 32, 773-781. https://doi.org/10.1016/j.exphem.2004.03.014

[17] Kacena, M.A., Todd, P. and Landis, W.J. (2004) Osteoblasts Subjected to Spaceflight and Simulated Space Shuttle Launch Conditions. In Vitro Cellular \& Developmental Biology-Animal, 39, 454-459. https://doi.org/10.1290/1543-706X(2003)039<0454:OSTSAS>2.0.CO;2

[18] Crawford-Young, S.J. (2006) Effects of Microgravity on Cell Cytoskeleton and Embryogenesis. The International Journal of Developmental Biology, 50, 183-191. https://doi.org/10.1387/ijdb.052077sc

[19] Schatten, H., Lewis, M.L. and Chakrabarti, A. (2001) Spaceflight and Clinorotation Cause Cytoskeleton and Mitochondria Changes and Increases in Apoptosis in Cultured Cells. Acta Astronautica, 49, 399-418. https://doi.org/10.1016/S0094-5765(01)00116-3

[20] Gershovich, P.M., Gershovich, J.G. and Buravkova, L.B. (2008) Simulated Microgravity Alters Actin Cytoskeleton and Integrin-Mediated Focal Adhesions of Cultured Human Mesenchymal Stromal Cells. Journal of Gravitational Physiology, 15, 203-204.

[21] Zayzafoon, M., Gathings, W.E. and McDonald, J.M. (2004) Modeled Microgravity Inhibits Osteogenic Differentiation of Human Mesenchymal Stem Cells and Increases Adipogenesis. Endocrinology, 145, 2421-2432.

https://doi.org/10.1210/en.2003-1156

[22] Meyers, V.E., Zayzafoon, M., Gonda, S.R., Gathings, W.E. and McDonald, J.M. (2004) Modeled Microgravity Disrupts Collagen I/Integrin Signaling during Osteoblastic Differentiation of Human Mesenchymal Stem Cells. Journal of Cellular Biochemistry, 93, 697-707. https://doi.org/10.1002/jcb.20229

[23] Meyers, V.E., Zayzafoon, M., Douglas, J.T. and McDonald, J.M. (2005) RhoA and Cytoskeletal Disruption Mediate Reduced Osteoblastogenesis and Enhanced Adipogenesis of Human Mesenchymal Stem Cells in Modeled Microgravity. Journal of Bone and Mineral Research, 20, 1858-1866. https://doi.org/10.1359/JBMR.050611

[24] Dai, Z.Q., Wang, R., Ling, S.K., Wan, Y.M. and Li, Y.H. (2007) Simulated Microgravity Inhibits the Proliferation and Osteogenesis of Rat Bone Marrow Mesen- 
chymal Stem Cells. Cell Proliferation, 40, 671-684. https://doi.org/10.1111/j.1365-2184.2007.00461.x

[25] Patel, M.J., Liu, W., Sykes, M.C., Ward, N.E., Risin, S.A., Risin, D. and Jo, H. (2007) Identification of Mechanosensitive Genes in Osteoblasts by Comparative Microarray Studies Using the Rotating Wall Vessel and the Random Positioning Machine. Journal of Cellular Biochemistry, 101, 587-599. https://doi.org/10.1002/jcb.21218

[26] Pan, Z., Yang, J., Guo, C., Shi, D., Shen, D., Zheng, Q., Chen, R., Xu, Y., Xi, Y. and Wang, J. (2008) Effects of Hindlimb Unloading on ex Vivo Growth and Osteogenic/Adipogenic Potentials of Bone Marrow Derived Mesenchymal Stem Cells in Rats. Stem Cells and Development, 17, 795-804. https://doi.org/10.1089/scd.2008.0254

[27] Ogneva, I.V. and Biryukov, N.S. (2013) Mathematical Modeling Cardiomyocyte's and Skeletal Muscle Fiber's Membrane: Interaction with External Mechanical Field. Applied Mathematics, 4, 1-6. https://doi.org/10.4236/am.2013.48A001

[28] Ogneva, I.V., Biryukov, N.S., Leinsoo, T.A. and Larina, I.M. (2014) Possible Role of Non-Muscle Alpha-Actinins in Muscle Cell Mechanosensitivity. PLoS ONE, 9, e96395. https://doi.org/10.1371/journal.pone.0096395

[29] Ogneva, I.V. and Biryukov, N.S. (2016) Lecithin Prevents Cortical Cytoskeleton Reorganization in Rat Soleus Muscle Fibers under Short-Term Gravitational Disuse. PLoS ONE, 11, e0153650. https://doi.org/10.1371/journal.pone.0153650

[30] Ogneva, I.V., Maximova, M.V. and Larina, I.M. (2014) Structure of Cortical Cytoskeleton in Fibers of Mouse Muscle Cells after Being Exposed to a 30-Day Space Flight on Board the BION-M1 Biosatellite. Journal of Applied Physiology, 116, 1315-1323. https://doi.org/10.1152/japplphysiol.00134.2014

[31] Ogneva, I.V., Gnyubkin, V., Laroche, N., Maximova, M.V., Larina, I.M. and Vico, L. (2015) Structure of the Cortical Cytoskeleton in Fibers of Postural Muscles and Cardiomyocytes of Mice after 30-Day 2g-Centrifugation. Journal of Applied Physiology, 118, 613-623. https://doi.org/10.1152/japplphysiol.00812.2014

[32] Ogneva, I.V., Belyakin, S.N. and Sarantseva, S.V. (2016) The Development of Drosophila Melanogaster under Different Duration Space Flight and Subsequent Adaptation to Earth Gravity. PLOS ONE, 11, e0166885. https://doi.org/10.1371/journal.pone.0166885

[33] Nygaard, M., Kragelund, B.B., Papaleo, E. and Lindorf-Larsen, K. (2017) An Efficient Method for Estimating the Hydrodynamic Radius of Disordered Protein Conformations. Biophysical Journal, 113, 550-557. https://doi.org/10.1016/j.bpj.2017.06.042

[34] Neuman, K.C., Abbondanzieri, E.A., Landick, R., Gelles, J. and Block, S.M. (2003) Ubiquitous Transcriptional Pausing Is Independent of RNA Polymerase Backtracking. Cell, 115, 437-447. https://doi.org/10.1016/S0092-8674(03)00845-6

[35] Darzacq, X., Shav-Tal, Y., de Turris, V., Brody, Y., Shenoy, S.M., Phair, R.D. and Singer, R.H. (2007) In Vivo Dynamics of RNA Polymerase II transcription. Nature Structural \& Molecular Biology, 14, 796-806. https://doi.org/10.1038/nsmb1280

[36] Shyu, A.B., Greenberg, M.E. and Belasco, J.G. (1989) The c-Fos mRNA Is Targeted for Rapid Decay by Two Distinct mRNA Degradation Pathways. Genes \& Development, 3, 60-72. https://doi.org/10.1101/gad.3.1.60

[37] Palmiter, R.D. (1975) Quantitation of Parameters that Determine the Rate of Ovalbumin Synthesis. Cell, 4, 189-197. https://doi.org/10.1016/0092-8674(75)90167-1

[38] Princiotta, M.F., Finzi, D., Qian, S.B., Gibbs, J., Schuchmann, S., Buttgereit, F., Bennink, J.R. and Yewdell, J.W. (2003) Quantitating Protein Synthesis, Degradation, and Endogenous Antigen Processing. Immunity, 18, 343-354. 
https://doi.org/10.1016/S1074-7613(03)00051-7

[39] Morey-Holton, E., Globus, R.K., Kaplansky, A. and Durnova, G. (2005) The Hindlimb Unloading Rat Model: Literature Overview, Technique Update and Comparison with Space Flight Data. Advances in Space Biology and Medicine, 10, 7-40. https://doi.org/10.1016/S1569-2574(05)10002-1

[40] Lakhani, V., Tan, L., Mukherjee, S., Stewart, W.C.L., Swords, W.E. and Das, J. (2018) Mutations in Bacterial Genes Induce Unanticipated Changes in the Relationship between Bacterial Pathogens in Experimental Otitis Media. Royal Society Open Science, 5, Article ID: 180810. https://doi.org/10.1098/rsos.180810

[41] Goffart, S., Franko, A., Clemen, C.S. and Wiesner, R.J. (2006) Alpha-Actinin 4 and BAT1 Interaction with the Cytochrome $c$ Promoter Upon Skeletal Muscle Differentiation. Current Genetics, 49, 125-135. https://doi.org/10.1007/s00294-005-0043-0 